\title{
Estrategias didácticas para desarrollar prácticas inclusivas en docentes de educación básica
}

\author{
Didactic strategies to develop inclusive practices \\ in basic education teachers
}

\author{
Jessica PaOla Palacios-Garay* \\ Violeta Cadenillas-Albornoz** \\ Patricia Gladys Chávez-Ortiz*** \\ RUth Alina Flores-BarRIOs**** \\ Karol Molra Abad-Escalante*****
}

\section{Resumen}

Objetivo. Establecer la incidencia de las estrategias didácticas en el desarrollo de prácticas inclusivas en docentes de educación básica regular. Metodología. El presente estudio se enmarca en el paradigma positivista, de enfoque cuantitativo, tipo sustantivo, diseño no experimental y correlacional causal. Resultados. Los resultados evidencian bajos niveles del uso de estrategias didácticas para desarrollar prácticas inclusivas en docentes de educación básica. Conclusión. Las estrategias didácticas inciden en el desarrollo de las prácticas inclusivas en docentes de educación básica, puesto que la razón de verosimilitud del modelo de regresión logística ordinal es significativa p $<0,05$ y la incidencia es explicada en un 55,9\% de las variables predictoras: motivación, problematización, recojo de saberes previos, propósito de la sesión, gestión y acompañamiento, aplicación o transferencia y evaluación en el desarrollo de las prácticas inclusivas en docentes de educación básica.

Palabras clave: estrategias didácticas, prácticas inclusivas, estrategias de aprendizaje cooperativo, estrategias de aprendizaje dialógico, estrategias integrales

\section{Abstract}

Objective: To establish the incidence of didactic strategies in the development of inclusive practices in teachers of regular secondary education. Methodology: This study is framed in the positivist paradigm of quantitative focus, substantive type, non-experimental design, causal correlation. Results: The results show low levels of the use of didactic strategies to develop inclusive practices by secondary education teachers. Conclusion: Didactic strategies influence the development of inclusive practices in secondary education teachers since the authenticity ratio of the ordinal logistic regression model is significant $\mathrm{p}<0.05$, and the incidence is explained in a $55.9 \%$ of the predictor variables: motivation, problematization, collection of previous knowledge, purpose of the session, management and accompaniment, application or transfer and evaluation in the development of inclusive practices of secondary education teachers.

Key words: didactic strategies, inclusive practices, cooperative learning strategies, dialogic learning strategies, comprehensive strategies.

\footnotetext{
*Universidad Norbet Wiener. Lima, Perú. E-mail: jessica.palacios@uwiener.edu.pe. (1) orcid.org/0000-0002-2315-1683. Google Scholar

${ }^{* *}$ Universidad César Vallejo. Lima, Perú. E-mail: cadealbo@ucvvirtual.edu.pe. (1) orcid.org/0000-0002-4526-2309.

Google Scholar

***. Universidad César Vallejo. Lima, Perú. E-mail: pchavezort@ucvvirtual.edu.pe. (D) orcid.org/0000-0003-3791-7368.

Google Scholar

***** Universidad César Vallejo. Lima, Perú. E-mail: adarafb@gmail.com. (1) orcid.org/0000-0002-5361-3228.

Google Scholar

****** Universidad César Vallejo. Lima, Perú. E-mail: kabad@ucv.edu.pe. (1) orcid.org/0000-0003-3963-9883.
} 


\section{Introducción}

En los referentes teóricos se puede establecer que para el desarrollo de prácticas inclusivas de los docentes se requiere del desarrollo de estrategias didácticas las cuales deben estar configuradas desde el currículo. Al respecto, Rodríguez (2018) manifestó que las estrategias didácticas en una perspectiva teórico conceptual de educación inclusiva son uno de los factores que inciden en la práctica docente; asimismo, es importante tomar en cuenta la sistematización de las estrategias didácticas para el logro de la educación inclusiva en estudiantes que pertenecen a la diversidad. La sistematización de las estrategias toma como base la propuesta de Jara (1989), quien propuso cinco momentos para lograr la sistematización: (a) tomar en cuenta el punto de partida, (b) realizar preguntas iniciales, (c) recuperar procesos vividos, (d) reflexionar sobre temas de fondo, (e) tomar en cuenta los puntos de llegada que posibilitaron la generación de conocimientos, produciendo saberes sociales, y reconocer que los factores utilizados en la sistematización inciden y evitan la deserción escolar de los estudiantes con diversidad funcional y los impactos que tienen las estrategias didácticas para el logro de la inclusión efectiva en estudiantes que pertenecen a la diversidad o inclusividad.

Asimismo, García y Delgado (2017) plantearon que las estrategias didácticas que se implementen, deben tomar en cuenta la diversidad desde el currículum, así como la utilización y funcionalidad de las estrategias, señalando la importancia de las diferentes formas de organización espacial, técnicas y métodos usados. La tarea del docente es conocer, analizar e incluso aplicar lo que supone cuestionarnos la práctica educativa más allá del enfoque inclusivo.

Omaña y Alzolar (2017) propusieron que las estrategias pedagógicas o didácticas para la inclusión del estudiante con discapacidad son necesarias debido a la inadecuada atención de los docentes a los estudiantes inclusivos o de la diversidad; igualmente, detectó la necesidad de emplear estrategias para la inclusión y el diseño de actividades para la formación de los docentes hacia la inclusión. Estas actividades podrían ser por medio de un espacio virtual y orientado al uso estrategias didácticas adaptadas a la población perteneciente a la discapacidad o diversidad.

En cuanto a las prácticas inclusivas, Flores, García y Romero (2017) encontraron que los profesores presentan prácticas inclusivas; sin embargo, requieren mayor apoyo en las condiciones de mobiliario y estructura física del aula, adaptaciones de la metodología y mejorar la relación docente estudiante. Asimismo, se encontró la necesidad de planificar la actualización de los docentes, de tal manera que se enriquezcan conceptualmente y propicie la implementación de la educación inclusiva.

García, Romero, Rubio, Flores y Martínez (2015) compararon las prácticas inclusivas de docentes de servicios de educación especial y regular. Encontraron que las instituciones 
educativas regulares, según su auto-reporte, las trabajan de manera colaborativa con profesionales de educación especial y no siempre planifican tomando en cuenta las necesidades de los estudiantes inclusivos o pertenecientes a la diversidad; mientras los docentes de instituciones educativas especiales prefieren el trabajo independiente y los docentes tienen más prácticas inclusivas que los docentes de educación regular; lo cual parece explicarse por sus condiciones de trabajo, que favorece el número reducido de estudiantes por grupos, por cultura escolar y los conocimientos sobre discapacidad.

\section{Teoría humanista}

Rogers (1967) es el creador y promotor de terapias dirigidas a las personas. Comenzó a partir de la realidad que los humanos nacen con disposiciones positivas, con tendencia constructiva que quieren expresarse, pero las situaciones para esto deben cumplirse. La tendencia hacia la actualización es convertirse en lo que se llega al nacer, la persona es innata, lo que hace que cada persona sea excepcional; si esto no sucede, se deberá a las limitaciones del desarrollo. La base de desarrollar la personalidad es el yo, que se basa totalmente en estudios y, por lo tanto, es dinámico y cambiante. Un problema principal es el amor incondicional sobre la idea de que debe manifestarse hacia los niños, si ya no se siente aceptado, se verá obligado a generar comportamientos para que así sea; por lo tanto, surge la incongruencia. Toda persona tiene derecho al reconocimiento incondicional. La consideración del individuo en un ambiente completo, de relaciones interpersonales y emociones intrapersonales. Permite tener en cuenta al estudiante en todas sus dimensiones, desde un ángulo holístico, que integra al niño y su función activa en su estudio.

\section{Teoría de cognición social}

Bandura (1971) mencionó que las personas aprenden mediante las observaciones, las imitaciones y siguiendo el ejemplo de los demás; presenta el puente entre las teorías conductuales y cognitivas de aprendizaje, centrándose en la relevancia de la atención, la memoria y la motivación de los estudiantes. Por lo tanto, el aprendizaje a través de la observación, la imitación y ejemplo de otros, se asocia con la investigación realizada porque los estudiantes aprenden del ejemplo, del comentario, de lo que estudian, esto les permite tener una versión que se adapta rápidamente a su conducta. El uso de estrategias didácticas con cada caso de diversidad o inclusión, es parte de lo que propone esta teoría y, al igual que la anterior, la cognición es crítica para obtener conocimiento.

\section{Teoría de la autoeficacia como aprendizaje}

Para Bandura (1971) la autoeficacia es fundamental para el concepto de aprendizaje social cognitivo, ya que enfatiza la importancia de las creencias propias de las personas, 
aproximadamente su potencial para examinar algo nuevo. La idea sugiere que el sentimiento de poder propio influye en la voluntad de una persona para intentar hacer y analizar nuevos asuntos, por lo que se genera un esfuerzo y perseverancia con la intención de observar su conocimiento. Por lo tanto, tener autoeficacia es lo importante para el éxito del aprendizaje. Esta idea es de importancia excepcional dado que las personas con diversidad útil requieren de muchas imitaciones y ejemplos, por lo que la asistencia a las escuelas les permite tener tiempo suficiente para comentar y también reproducir a través de la imitación los movimientos de sus compañeros.

\section{Teoría del desarrollo cognoscitivo}

Piaget (1975) destacó que "el desarrollo cognoscitivo es un proceso de construcción de estructuras lógicas, explicados por mecanismos endógenos y en el cual la intervención social solo puede ser "facilitadora" u "obstaculizadora" en el proceso" (p. 56). Además, la inteligencia, al margen de su contenido y nivel de mejora, se define por componentes interdependientes: la institución y las adaptaciones que son invariables porque a través de la larga forma de mejora cognitiva que generalmente están presentes, de ninguna manera varían permitiendo la función intelectual y los procesos de adaptación. Por lo tanto, esta teoría es importante para el proceso de mejora intelectual de los estudiantes con una diversidad ya que, a través de las adaptaciones mentales, que son procesos que siempre están presentes en el conocimiento, se logra la mejora cognitiva esperada, algunos en un grado y otros en otros grados, dependiendo de la diversidad que ofrecen a su ritmo personal. En este principio, puede ver los tres aditivos de conocer: cognición, afectividad y comportamiento, que son muy importantes para el desarrollo de prácticas inclusivas.

\section{Teoría sociocultural del desarrollo}

Vygotsky (1979) propuso que el desarrollo social y cultural, por lo que pone énfasis en los mecanismos de influencia educativa, en los que la medición social de conocer es un tema esencial. Es una actuación de carácter individual, sin embargo, en este caso lo individual no se contrapone a lo social. Los estudiantes crean conocimiento y al mismo tiempo construye colectivamente con otros; la ayuda de los demás, el docente, los padres, los hermanos, los diferentes medios de comunicación, es fundamental para construir conocimiento. La educación es una práctica social, dentro de este marco se avanzan tácticas de socialización y creación. Por lo tanto, en esta mejora de la personalidad entra en juego la capacidad del maestro para atender las diferencias dentro del aula de la escuela, siendo un método multidireccional, donde todos los colaboradores investigan y con las pinturas corporativas el conocimiento adquirido toma región con los humanos con un rango útil, como con algún otro estudiante de diferente situación. 


\title{
Referente conceptual
}

\section{Estrategias didácticas}

Rosales (2004) definió las estrategias didácticas como el conjunto de actividades que el maestro realiza con intenciones claras y explícitamente pedagógicas, y presenta tres niveles: pre activo, interactivo y pos activo. Esta teoría atribuye al profesor una importancia decisiva como guía y facilitador de los aprendizajes, considerando el correcto accionar del docente.

Rajadell (2002) y Minedu (2019) establecieron que el enfoque de intervención didáctica sucede en un período de tiempo, y se definió como una acción secuencial sin duda consciente del profesional en educación, guiado por uno o más principios de didáctica, y que se encamina a la optimización de las formas de enseñar y estudiar, de enseñanza-aprendizaje.

Santos y Portaluppi (2011), explicaron que las estrategias didácticas inclusivas son "la puerta de entrada hacia el pleno ejercicio de los derechos que todos los seres humanos poseen. Constituye un elemento de humanización y sensibilización frente a las diferentes realidades que se presentan en el contexto educativo y social” (p. 102).

\section{Procesos pedagógicos en la sección de aprendizaje}

En cuanto a los procesos pedagógicos, Coto (2005) afirmó que:

\begin{abstract}
Son actividades que desarrolla el docente de manera intencional con el objeto de mediar en el aprendizaje del estudiante. Estas prácticas docentes son un conjunto de acciones intersubjetivas y saberes que acontecen entre los que participan en el proceso educativo con la finalidad de construir conocimientos, clarificar valores y desarrollar competencias para la vida en común. Cabe señalar que los procesos pedagógicos no son momentos, son recurrentes y se acuden a ellos en cualquier momento que sea necesario. (p. 40)
\end{abstract}

Por tanto, se entiende que los procesos pedagógicos se encuentran sustentados teóricamente; a su vez en Coto (2005) manifestó que son el conjunto de eventos, interacciones e intercambios que suceden en los procesos de enseñanza-aprendizaje, procesos que suceden dentro o fuera de las aulas, donde el docente está encaminado a desarrollar las capacidades y por ende las competencias en los estudiantes, por lo que es importante que en este proceso tomen en cuenta a los estudiantes inclusivos o pertenecientes a la diversidad, con la finalidad de que los estudiantes puedan ser competentes y afronten su realidad y, de esta forma, construyan sus propios aprendizajes. 


\section{Dimensión 1: Problematización}

Son situaciones que presentan retos o desafíos, problemas o dificultades que pueden partir del contexto, de los intereses, necesidades y expectativas de los estudiantes. Ponen a prueba las competencias y capacidades que planifica el docente para resolverlo y en esta planificación es importante que el docente tome en cuenta las prácticas inclusivas para que los estudiantes puedan poner a prueba sus capacidades durante la problematización.

\section{Dimensión 2: Propósito}

El propósito implica informar o dar a conocer a los estudiantes las competencias, capacidades y desempeños que deben alcanzar los estudiantes, los aprendizajes que se espera que logren al finalizar la sesión de clase, los tipos de actividades que realizarán y cómo serán evaluados. Este propósito debe expresar la intensión de aprendizaje y las prácticas inclusivas que desarrollará el docente con la finalidad de involucrar a todos los estudiantes como parte sus prácticas inclusivas.

\section{Dimensión 3: Motivación}

La auténtica motivación incita y provoca a los estudiantes, los invita a perseverar en las resoluciones de los desafíos con voluntad y expectativas hasta el final de los procesos, en esta etapa es importante la despenalización del error, especialmente para que los estudiantes inclusivos o pertenecientes a la diversidad se animen e intenten resolver los desafíos.

Así mismo, señaló que la motivación es promoción o estímulo en los estudiantes o el grupo de estudiantes, con el interés por aprender, a través de estímulos seguros, y con la creación de las condiciones corporales y psicológicas relevantes. Puede hacer preguntas que generen diálogo, un póster, una reflexión, presentación de objetos reales, presentación de fotos alusivas al tema, anécdotas, entre otros y de este modo captar la atención de todos, cerrando las brechas de la inclusión.

\section{Dimensión 4: Saberes previos}

La función que ejerce el acopio de los saberes previos es pedagógica, pues sirven para vincular los saberes previos con los saberes próximos y potenciales, además de que es muy útil para tomar decisiones sobre la planificación curricular. Es importante recordar que se deben preparar estrategias adecuadas para garantizar la participación de los estudiantes inclusivos o pertenecientes a la diversidad 


\section{Dimensión 5: Gestión y acompañamiento del aprendizaje}

La gestión de generación del conocimiento consiste en la anticipación de estrategias a utilizar durante los procesos de enseñanza aprendizaje, estas generan interacciones docente estudiante, estudiantes y estudiantes de calidad, para planificar las estrategias adecuadas como parte de las prácticas inclusivas. Es muy importante tomar en cuenta la retroalimentación durante todo el proceso y finalización de las actividades.

\section{Dimensión 6: Aplicación o transferencia de la información}

En la transferencia de la información, los estudiantes deben ejecutar las capacidades en situaciones nuevas, donde ponen en práctica las teorías y conceptualizaciones adquiridas. Durante esta etapa el docente debe planificar prácticas inclusivas que garanticen la participación de los estudiantes inclusivos o de la diversidad, debido a que es el momento cumbre de la sistematización de los aprendizajes.

\section{Dimensión 7: Evaluación}

La etapa de la evaluación se refiere a la recolección frecuente de información sobre el progreso de los aprendizajes y así asegurar evaluación flexible, abierta y cíclica. La evaluación de los procesos de aprendizaje debe ser formativa, de tal manera que se pueda recoger información relevante para la sistematización de la planificación y se puedan hacer reajustes en las siguientes sesiones.

\section{Prácticas inclusivas}

Echeita (2014) definió a la educación inclusiva como técnica estratégica diseñada para facilitar el aprendizaje del nuevo conocimiento de todos los involucrados dentro del proceso académico, los lleva al éxito y a la mejora de su calidad de vida. Dado que el objetivo de educación inclusiva apunta a disminuir cualquier forma de exclusión y marginación a través de los deseos comunes y la participación legítima, los estudiantes pueden contribuir y recibir lecciones de manera regular y ser estudiantes activos con sus compañeros de aula.

Martínez (2014) afirmó que:

La educación inclusiva debe ser entendida como la posibilidad de construir procesos que den respuesta a las necesidades de todos los estudiantes que integran una institución educativa. La finalidad es lograr que todos los educandos tengan las mismas oportunidades de aprender evitando la exclusión de aquellos considerados por parte de la educación tradicional como diferentes. (p. 76) 
En ese sentido, la organización del sistema educativo como espacio al cual todos acceden representa el primer paso de la consolidación de una sociedad diferente. Es necesaria la comprensión de que la diversidad es una realidad representada en cada uno de los ámbitos en los cuales las personas se desenvuelven y la educación no es ajena a estas circunstancias.

Para García y Delgado (2017) reconocer que existen estudiantes con necesidades educativas que les impide aprender en las mismas condiciones que los demás compañeros, "permitirá a los docentes ajustar los currículos e implementar estrategias que favorezcan a la población escolar y contribuyan a la formación integral" (p. 10). En educación se evidencia que los estudiantes requieren apoyo pedagógico para ayudarlos a ampliar sus competencias; por lo tanto, se deben realizar actividades que permitan a los estudiantes expresar libremente con la ayuda del maestro y contribuir al trabajo colaborativo.

Correa (2008) indicó que las prácticas educativas reflejan la cultura de la escuela y las políticas inclusivas; llevarlos a cabo posibilita al grupo desarrollar y mejorar la satisfacción de la educación que brindan. Sin embargo, las prácticas implican un sistema de mejora debido a que, mientras se implementan, se adaptan de acuerdo con las necesidades que se pueden proporcionar, permiten el advenimiento de técnicas, técnicas y estrategias didácticas, hasta que formen parte de estilos ordinarios de vida.

Además, la inclusión reconoce, por un lado, la variedad de fuentes y recursos de personas y, por otro, las capacidades de la sociedad para convertirlas en recursos en funcionamiento. Con la operación, se refiere a los asuntos que la persona logra ser o hacer cuando vive. Además, las prácticas inclusivas implican resultados, la información de las condiciones y la búsqueda de tácticas que se ejecutan en la dirección de la equidad, la justicia, la cooperación y las posibilidades idénticas. Para lo anterior, el trabajo colaborativo es el enfoque principal y la creación de alianzas una modalidad efectiva del mismo.

\section{Dimensión 1: Estrategias de aprendizaje cooperativo}

Santos, Lorenzo y Priegue (2009) afirmaron que el aprendizaje cooperativo es "una metodología educativa innovadora con el potencial necesario para cambiar las prácticas pedagógicas en las escuelas. Se basa en un enfoque constructivista que hace equilibrar a todos los participantes respecto a sus roles” (p. 289). Por lo tanto, la clave para garantizar los aprendizajes satisfactorios radica las interacciones con el medio y las personas que los rodean.

De igual manera, León, Felipe, Iglesias y Marugán (2014) afirmaron que "el aprendizaje cooperativo es una metodología eficaz que favorece una actitud crítica y de tolerancia, desarrollando cooperación, solidaridad y trabajo en equipo” (p. 414). 
La utilización del aprendizaje cooperativo representa un recurso eficaz para el fomento de la educación inclusiva, basada en interacciones entre los estudiantes, entre iguales. Este enfoque o práctica inclusiva considera la construcción de los aprendizajes desde situaciones consideradas reales, donde los estudiantes de diferentes disciplinas comparten protagonismo en la construcción del producto final, del que todos se sienten responsables, incluyendo a los estudiantes pertenecientes a la diversidad o inclusión (Navarro, González, López y Botella, 2015).

\section{Dimensión 2: Estrategias de aprendizaje dialógico}

Aubert, Flecha, García, Flecha y Racionero (2008) manifestaron que el conocimiento dialógico establece que se aprende a través de la interacción de la red con otros estudiantes, produciendo acuerdos intersubjetivos para muchos de los individuos. Lo esencial en su definición es que para el aprendizaje se debe analizar una gama masiva de interacciones y tan numerosas como sea posible, apoyando el diálogo en una datación de igualdad y ahora no de poder; esto significa que cualquiera tiene experiencia para hacer una contribución, por lo tanto, reconocer inteligencia cultural de todos los seres humanos. De acuerdo con el pensamiento dialógico de aprendizaje, el factor más crucial es la interacción que tiene lugar entre las personas, más allá de escuchar, analizar o escribir, aunque este último no las prescinde; sin embargo, las utiliza como enfoque.

Al respecto, Álvarez y González (2013) mencionaron que el conocimiento dialógico considera que los involucrados podrían hacer contribuciones relevantes a los procesos de enseñanzaaprendizaje (debido a la inteligencia cultural) y que, a su vez, todos se benefician de los procesos comunicativos que se desarrollan. En este contexto, los estudiantes que adquieren conocimientos y presentan dificultades o necesidades educativas especiales también se ven doblemente beneficiados: los procedimientos de procesos educativos les brindan redes para reconocer mejor el contenido curricular esperado para su etapa de instrucción y, al mismo tiempo, también pueden contribuir, aumentando su autoestima mediante la enseñanza de sus conocimientos a los demás.

\section{Dimensión 3: Estrategias de aprendizaje servicio y comunitario}

Batllé (2013) sostuvo que el aprendizaje de servicio y comunitario es un cóctel que mezcla los dos ingredientes que dan nombre a la herramienta, una mezcla con base en una propuesta didáctica experiencial orientada desde las premisas de "aprender haciendo".

García y Gavira (2014) afirmaron que el aprendizaje comunitario "es compromiso, porque supone una práctica que se proyecta desde la emoción” (p. 17), además, celebran las diferencias en la convivencia cooperativa que los articula. Así mismo, Batllé (2013) precisó que el aprendizaje en servicio y comunitario representa una filosofía que reconcilia la dimensión 
cognitiva y la dimensión ética de las personas; una pedagogía que reconcilia la calidad educativa e inclusión. Así, compromisos, emociones y ética se constituyen como la base de estas propuestas pedagógicas que promueven un aprendizaje experiencial, contextualizado y significativo, volcado en la acción para la comunidad.

\section{Dimensión 4: Estrategias para fomentar la participación social}

Moliner, Sanahuja y Benet (2017) manifestaron que cuando un estudiante se incorpora a un equipo es fundamental el establecimiento de una relación basada en respeto que sitúe a cada integrante del equipo ante los demás. Dentro de las actividades de acogida está la entrega de información a los nuevos que contribuyan a situarse rápidamente en el contexto de clase (responsabilidad, norma, espacios) y del centro.

Entre las estrategias que pueden ayudar a crear un ambiente social inclusivo se encuentran: (a) sistema de compañeros y amigos, es un tipo de red de apoyo que se basa en la creación de un círculo de amigos voluntarios que actúan como compañeros y amigos de alumnos con serias dificultades en la relación y adaptación (por muy diversos motivos); y, (b) círculo de amigos, consiste en su establecimiento alrededor de un alumno con necesidades educativas profundas a su llegada al centro, pretendiendo no solo prestar ayuda a la persona, sino hacer conscientes a los otros de la necesidad de ayuda de algunas personas, y el compromiso con ellas; (c) comisiones de apoyo entre compañeros, consiste en la creación dentro del aula de una comisión de compañeros a la que se van incorporando por turnos los distintos alumnos, y cuyo sentido es el análisis de las formas en que puede mejorarse el apoyo mutuo en el aula para que la misma sea una comunidad más acogedora; $y$, (d) estudiante ayudante, a través de una sencilla dinámica el grupo de estudiantes de una clase seleccionan a dos o tres de sus compañeros/ as por sus cualidades de ayuda a los demás y confianza, su capacidad de escuchar y analizar conflictos (Torrego, 2006).

\section{Dimensión 5: Estrategias integrales}

Moliner et al. (2017) denominaron así a aquellas estrategias de respuesta a la diversidad del aula que contemplan múltiples dominios de los procesos de enseñanza-aprendizaje. No deben apreciarse solo desde aspectos concretos como agrupación de estudiantes, todos los recursos físicos, temporales, materiales o de la selección de la metodología apropiada.

Las estrategias integrales deben ser tomadas en cuenta para desarrollar prácticas inclusivas en educación por los beneficios que presenta. Entre las principales estrategias integrales se encuentran: (a) los rincones y talleres, estos rincones suponen una gran oportunidad de desarrollo de práctica inclusiva de la enseñanza del docente, esta acoge principalmente a la diversidad e inclusión, no solo como una dimensión para conseguir el respeto del colectivo 
humano, sino de la propia enseñanza; los talleres están centrados en contenidos, procedimientos y exigencias de las actividades directas, tienen de por sí un gran potencial para responder a las necesidades que la inclusión o diversidad de un aula requieren; (b) los proyectos de trabajo inclusivos representan una estrategia de aprendizaje compartido, se ajustan en su filosofía de estrategia didáctica y de organización al currículum, una estrategia de enseñanza-aprendizaje, que afecta a las estructuras organizativas y metodológicas, y que organizan los contenidos desde las perspectivas globalizadoras (partiendo de la realidad global de los estudiantes, que son el objeto de los aprendizajes); y (c) la enseñanza compartida, donde la presencia de dos docentes dentro del aula mejora todas las posibilidades de atención de un alumnado heterogéneo, pero además inducen efectos añadidos a las propias razones o ventajas directas, como es la generación de un clima más abierto, más comunicativo dentro del aula, rompen con los esquemas del silencio y la homogeneidad que no favorece a los estudiantes inclusivos o de la diversidad, y lo que es más innovador e importante, a saber, que los docentes de apoyo pueden dejar de ser un elemento de ayuda a las dificultades de los aprendizajes de unos pocos, y pasan a ser un factor de calidad de los procesos de aula que benefician a una clase diversa en su conjunto, como es la realidad en nuestros días.

\section{Metodología}

Las instituciones educativas deben garantizar que la planificación individualizada mediante prácticas inclusivas garantice la mejora de los aprendizajes de los estudiantes inclusivos, los pertenecientes a la diversidad y todos ellos con necesidades educativas especiales; por lo que la presente investigación se enmarca dentro de un paradigma positivista Ricoy (como se citó en Ramos, 2015) establece que este paradigma es calificado de cuantitativo, empírico analista, racional y científico. En cuanto al enfoque cuantitativo Hernández y Mendoza (2018) establecieron que consiste en la demostración numérica de resultados. Por otro lado, el método considerado fue el hipotético-deductivo que consiste en la precisión de la problemática, el planteamiento de hipótesis y la prueba de las hipótesis para la emisión de las conclusiones del estudio (Bernal, 2010). El diseño de la investigación fue no experimental y según Hernández y Mendoza (2018) este tipo de estudio se realiza sin manipulación de las variables y observando los fenómenos en su contexto. La población del presente estudio estuvo constituida por 48 docentes de dos instituciones educativas públicas con atención a estudiantes considerados inclusivos y de la diversidad. En cuanto a la técnica utilizada para las variables están la observación, los instrumentos y la lista de cotejo. La variable estrategias didácticas contó con siete dimensiones con 26 ítems, mientras que la variable prácticas inclusivas contó con un instrumento de 24 ítems; ambos medidos con una escala ordinal de tipo Likert. 


\section{Resultados}

En la investigación se expresan los resultados, el análisis descriptivo de las variables y el análisis estadístico con las correspondientes interpretaciones de los datos obtenidos.

\section{Resultados del análisis descriptivo}

Tabla 1. Descriptivos de la variable estrategias didácticas.

\begin{tabular}{llrrrr}
\hline & & & & \multicolumn{2}{c}{$\begin{array}{c}\text { Porcentaje } \\
\text { acumulado }\end{array}$} \\
\hline Válido & Frecuencia & Porcentaje & Porcentaje válido & 16,7 \\
& Medio & 8 & 16,7 & 16,7 & 100,0 \\
& Total & 40 & 83,3 & 83,3 & \\
\hline
\end{tabular}

Fuente: base de datos.

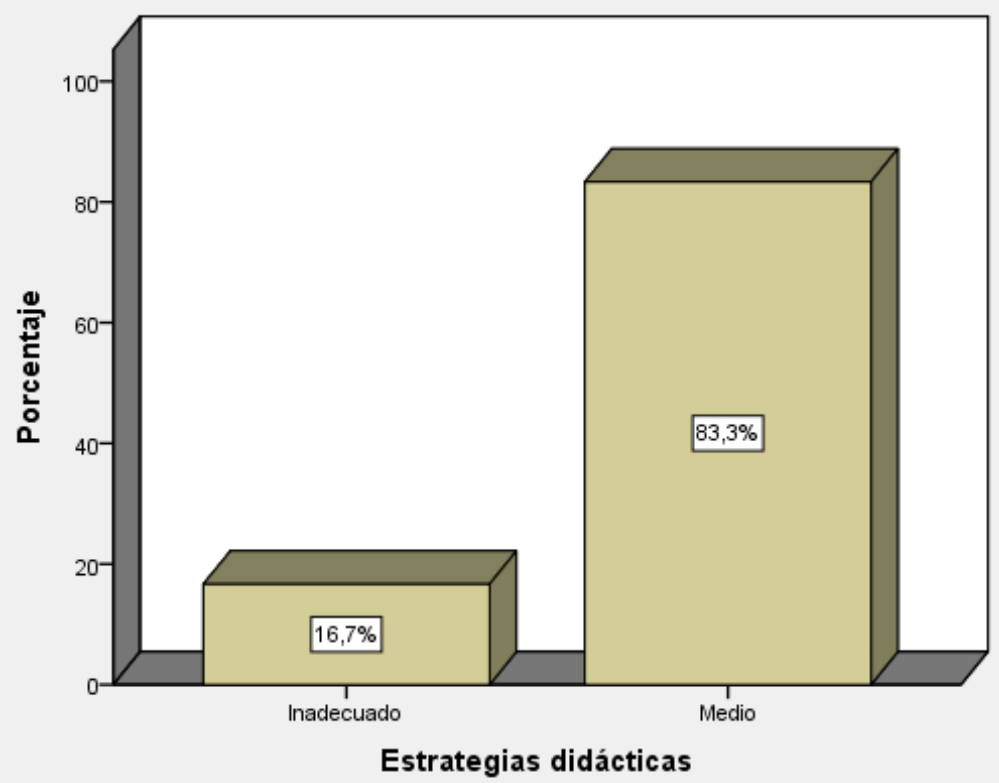

Figura 1. Descriptivos de la variable desarrollo de estrategias didácticas.

Fuente: base de datos. 
En la tabla 1 y figura 1 se evidenció que, en cuanto a las estrategias didácticas realizadas por los docentes, el 16,7\% mostró un nivel inadecuado de estrategias didácticas, el 83,3\% mostró un nivel medio de estrategias didácticas y ningún docente alcanzó el nivel adecuado de estrategias didácticas en educación básica.

Tabla 2. Descriptivos de la variable desarrollo de prácticas inclusivas.

\begin{tabular}{llrrrr}
\hline & & & & \multicolumn{2}{c}{$\begin{array}{c}\text { Porcentaje } \\
\text { acumulado }\end{array}$} \\
\hline Válido & Bajo & 23 & 47,9 & 47,9 & 47,9 \\
& Regular & 12 & 25,0 & 25,0 & 72,9 \\
& Alto & 13 & 27,1 & 27,1 & 100,0 \\
& Total & 48 & 100,0 & 100,0 & \\
\hline
\end{tabular}

Fuente: base de datos.

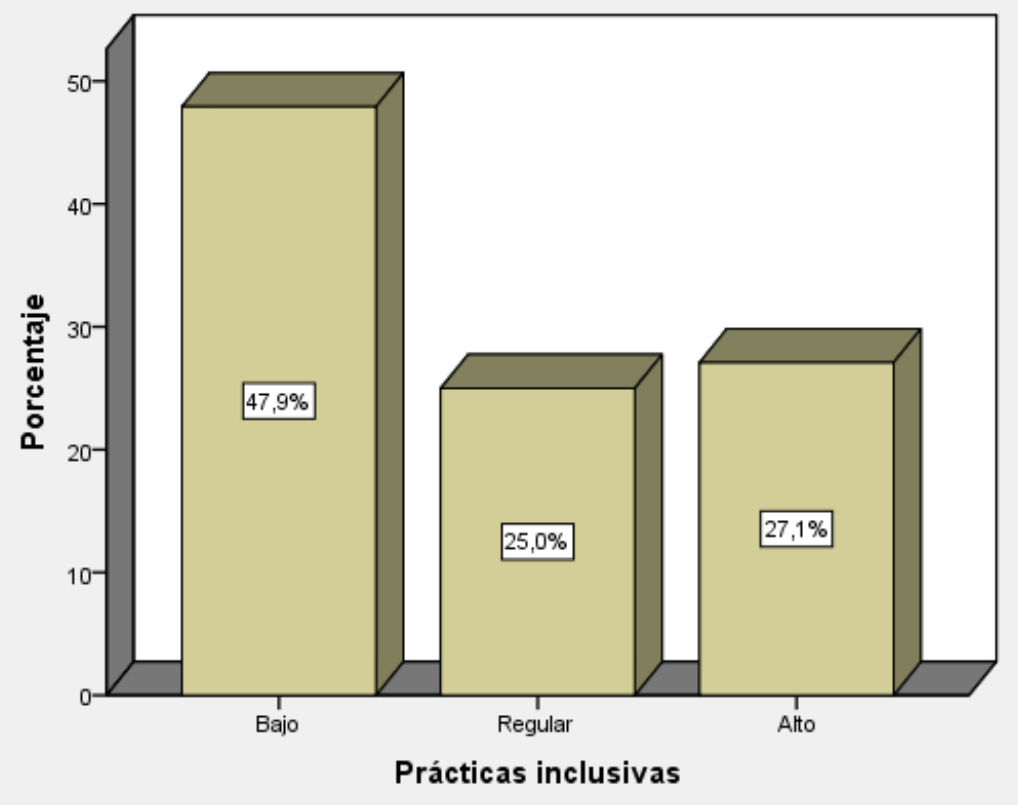

Figura 2. Descriptivos de la variable desarrollo de prácticas inclusivas.

Fuente: base de datos. 
En la tabla 2 y figura 2 se evidenció que, en cuanto a las prácticas inclusivas realizadas por los docentes, el 47,9\% mostró un nivel bajo de prácticas inclusivas, el 27,1\% mostró un nivel alto y el 25\% mostró un nivel regular de prácticas inclusivas y el desarrollo de prácticas inclusivas en educación básica.

\section{Resultados del análisis inferencial}

Tabla 3. Información del ajuste del modelo, que explica cómo las estrategias didácticas inciden en el desarrollo de las prácticas inclusivas en docentes de educación básica.

\begin{tabular}{|c|c|c|c|c|}
\hline \multicolumn{5}{|c|}{ Información de ajuste de los modelos } \\
\hline Modelo & $\begin{array}{l}\text { Logaritmo de la } \\
\text { verosimilitud - } 2\end{array}$ & Chi-cuadrado & gl & Sig. \\
\hline Sólo intersección & 98,879 & & & \\
\hline Final & 66,495 & 32,384 & 13 & ,002 \\
\hline Función de enlace: Logit. & & & & \\
\hline
\end{tabular}

Fuente: base de datos.

En la tabla 3 se evidencia que la prueba de ajuste de modelo o de contraste, la razón de verosimilitud es significativo $\left(\mathrm{x}^{2}=32,384 ; \mathrm{p}<0,05\right)$ interpretándose que las estrategias didácticas inciden en el desarrollo de las prácticas inclusivas en docentes de educación básica, por lo que los docentes deben planificar y desarrollar cada una de las estrategias didácticas con claridad y precisión para que puedan desarrollar prácticas inclusivas.

Tabla 4. Bondad de ajuste del modelo, que explica cómo las estrategias didácticas inciden en el desarrollo de las prácticas inclusivas en docentes de educación básica.

\begin{tabular}{lrrrr}
\hline \multicolumn{2}{c}{ Bondad de ajuste } & & \\
\hline & Chi-cuadrado & gl & Sig. \\
\hline Pearson & 163,801 & 45 & &, 000 \\
Desvianza & 64,873 & 45 & &, 028 \\
Función de enlace: Logit. & & & & \\
\hline
\end{tabular}

Fuente: base de datos.

En la tabla 4 se evidencia que la bondad de ajuste o prueba de regresión comprueba la calidad en el ajuste de los valores prestablecidos por el modelo evidenciado. La desviación $\left(\mathrm{x}^{2}=\right.$ 64,873 ) muestra una $\mathrm{p}<0,05$, estableciéndose que el modelo consideró que las estrategias didácticas inciden en el desarrollo de las prácticas inclusivas en docentes de educación básica, siendo válido y aceptado. 
Tabla 5. Pseudo $\mathrm{R}^{2}$ del modelo, que explica cómo las estrategias didácticas inciden en el desarrollo de las prácticas inclusivas en docentes de educación básica.

\begin{tabular}{lr}
\hline & Pseudo R cuadrado \\
\hline Cox y Snell &, 491 \\
Nagelkerke &, 559 \\
McFadden &, 320 \\
Función de enlace: Logit. & \\
\hline
\end{tabular}

Fuente: base de datos.

En la tabla 5 se evidencia que el valor de Cox y Snell explican una variabilidad de los datos en ,491 o 49,1\%, por lo que al convertir en $\mathrm{R}^{2}$ Nagelkerke obtuvo un valor de ,559 significando que el modelo puede explicar en un 55,9\% la variabilidad de la data, y el resultado de McFadden obtuvo ,320, lo que demuestra que la data se ajusta en 32\% y el 44,1\% es explicado por diversas variables que no forman parte de la investigación.

Tabla 6. Estimaciones del parámetro que explica cómo las estrategias didácticas inciden en el desarrollo de las prácticas inclusivas en docentes de educación básica.

\begin{tabular}{|c|c|c|c|c|c|c|c|c|}
\hline \multicolumn{9}{|c|}{ Estimaciones de parámetro } \\
\hline & & \multirow[b]{2}{*}{ Estimación } & \multirow[b]{2}{*}{$\begin{array}{c}\text { Error } \\
\text { estándar }\end{array}$} & \multirow[b]{2}{*}{ Wald } & \multirow[b]{2}{*}{$\mathrm{gl}$} & \multirow[b]{2}{*}{ Sig. } & \multicolumn{2}{|c|}{ Intervalo de confianza al 95\% } \\
\hline & & & & & & & $\begin{array}{l}\text { Límite } \\
\text { inferior }\end{array}$ & $\begin{array}{c}\text { Límite } \\
\text { superior }\end{array}$ \\
\hline \multirow[t]{2}{*}{ Umbral } & {$[$ Prac_inclu $=1]$} & $-25,108$ & 3,182 & 62,276 & 1 & ,000 & $-31,344$ & $-18,872$ \\
\hline & {$[$ Prac_inclu $=2]$} & $-23,179$ & 3,131 & 54,805 & 1 & ,000 & $-29,316$ & $-17,042$ \\
\hline \multirow[t]{20}{*}{ Ubicación } & {$[$ Motiv $=1]$} & $-18,537$ & ,958 & 374,705 & 1 & ,000 & $-20,414$ & $-16,660$ \\
\hline & {$[$ Motiv $=2]$} & $-20,787$ &, 000 & . & 1 & . & $-20,787$ & $-20,787$ \\
\hline & {$[$ Motiv $=3]$} & $0^{\mathrm{a}}$ & . & . & 0 & . & . & \\
\hline & {$[$ Proble $=1]$} &,- 992 & 2,518 &, 155 & 1 & ,693 & $-5,928$ & 3,943 \\
\hline & {$[$ Proble $=2]$} &,- 477 & 2,265 &, 044 & 1 & ,833 & $-4,916$ & 3,962 \\
\hline & {$[$ Proble $=3]$} & $0^{\mathrm{a}}$ & . & . & 0 & . & . & \\
\hline & {$[$ Sab_prev $=1]$} & $-1,388$ & 1,056 & 1,727 & 1 &, 189 & $-3,457$ & ,682 \\
\hline & {$[$ Sab_prev $=2]$} & $0^{\mathrm{a}}$ & . & . & 0 & . & . & \\
\hline & [Propó=1] & $-1,824$ & 1,935 & ,888 & 1 & ,346 & $-5,617$ & 1,969 \\
\hline & [Propó=2] & $-2,070$ & 1,843 & 1,261 & 1 & ,261 & $-5,683$ & 1,543 \\
\hline & [Propó=3] & $0^{\mathrm{a}}$ & . & . & 0 & . & . & \\
\hline & [Ges_Acom $=1]$ & 1,013 & 1,832 & ,306 & 1 &, 580 & $-2,578$ & 4,604 \\
\hline & [Ges_Acom=2] &,- 704 & 1,533 & ,211 & 1 & ,646 & $-3,709$ & 2,301 \\
\hline & [Ges_Acom=3] & $0^{\mathrm{a}}$ & . & . & 0 & . & . & \\
\hline & [Aplic_transf $=1$ ] & ,425 & 1,708 & ,062 & 1 &, 803 & $-2,923$ & 3,773 \\
\hline & [Aplic_transf $=2$ ] & , 546 & 1,374 & , 158 & 1 & ,691 & $-2,147$ & 3,240 \\
\hline & [Aplic_transf $=3$ ] & $0^{\mathrm{a}}$ & . & . & 0 & . & . & \\
\hline & {$[$ Eval=1] } & $-4,675$ & 1,557 & 9,022 & 1 & ,003 & $-7,726$ & $-1,624$ \\
\hline & {$[$ Eval=2] } & $-2,832$ & 1,326 & 4,562 & 1 & ,033 & $-5,431$ &,- 233 \\
\hline & {$[$ Eval=3] } & $0^{\mathrm{a}}$ & . & . & 0 & . & . & \\
\hline
\end{tabular}

Función de enlace: Logit.

a. Este parámetro está establecido en cero porque es redundante.

Fuente: base de datos. 
En la tabla 6 se evidencia que la evaluación de las estrategias didácticas (Wald=9,906) presentó $\mathrm{p}=0,003(\mathrm{p}<0,05)$ y predicen mejor el desarrollo de las prácticas inclusivas, en docentes de educación básica.

\section{Discusión}

De acuerdo al objetivo general de la investigación, se encontró que las estrategias didácticas inciden en el desarrollo de las prácticas inclusivas en docentes de educación básica, puesto que $\mathrm{p}<0,05$ y la incidencia es explicada en un 55,9\% de incidencia de los factores predictores: motivación, problematización o contextualización, acopio de saberes previos, propósito de la sesión, gestión y acompañamiento del aprendizaje, aplicación o transferencia de la información y evaluación en el desarrollo de las prácticas inclusivas en docentes de educación básica. Estos resultados coinciden con diversos estudios y, al respecto, Rodríguez (2018) manifestó que las estrategias didácticas en una perspectiva teórico conceptual de educación inclusiva es uno de los factores que inciden en la práctica docente y es importante tomar en cuenta la sistematización de las estrategias didácticas para el logro de la educación inclusiva en estudiantes que pertenecen a la diversidad; estas estrategias didácticas producen saberes cognitivos y sociales, además de reconocer que los factores que contemplan las estrategias didácticas inciden y evitan la deserción escolar de los estudiantes con diversidad o inclusión.

En la misma línea del pensamiento, García y Delgado (2017) plantean que las estrategias didácticas que se implementen deben tomar en cuenta la diversidad desde el currículum, así como la utilización y funcionalidad de las estrategias, señalando la importancia de las diferentes formas de organización espacial, técnicas y métodos usados. Es la tarea del docente conocer, analizar e incluso la aplicación, y supone cuestionarnos la práctica educativa más allá del enfoque inclusivo.

Es importante subrayar que la atención a los estudiantes inclusivos y pertenecientes a la diversidad es necesaria para cerrar las brechas de la inclusión que todavía se evidencian, tal como se encontró en el presente estudio, obteniéndose que, en cuanto a las estrategias didácticas realizadas por los docentes, el 16,7\% mostró un nivel inadecuado de estrategias didácticas, el 83,3\% mostró un nivel medio de estrategias didácticas y ningún docente alcanzó el nivel adecuado de estrategias didácticas en docentes de educación básica y el 47,9\% mostró un nivel bajo de prácticas inclusivas, el 27,1\% mostró un nivel alto y el 25\% mostró un nivel regular de prácticas inclusivas y el desarrollo de prácticas inclusivas en docentes de educación básica. Coincidiendo con Omaña y Alzolar (2017) encontraron una inadecuada atención de los docentes a los estudiantes inclusivos o de la diversidad, asimismo, detectó la necesidad de emplear estrategias para la inclusión y el diseño de actividades para la formación de los docentes hacia la inclusión. Estas actividades deben orientarse al uso de estrategias didácticas adaptadas a la población perteneciente a la discapacidad o diversidad y en cuanto a las prácticas 
inclusivas. Flores et al. (2017) encontraron que los profesores presentan prácticas inclusivas; sin embargo, requieren mayor apoyo en las condiciones de mobiliario y estructura física del aula, adaptaciones de la metodología y mejorar la relación docente estudiante; asimismo, se encontró la necesidad de planificar la actualización de los docentes, de tal manera que enriquezcan conceptualmente a los docentes y propicie la implementación de la educación inclusiva. Es una prioridad la atención a la problemática y al respecto, Molbaek (2018) manifestó que la inclusión tiene una prioridad en las políticas de educación del mundo y los docentes deben realizar prácticas inclusivas e incrementar la atención a la diversidad de los estudiantes y mejorar las condiciones ambientales del aprendizaje. Al mismo tiempo, Tichá \& Abery (2018) indicaron que las estrategias y prácticas inclusivas han demostrado apoyo y garantiza la educación en estudiantes inclusivos o de la diversidad.

\section{Conclusiones}

En relación al objetivo planteado se encontró que las estrategias didácticas inciden en el desarrollo de las prácticas inclusivas en docentes de educación básica, puesto que la razón de verosimilitud del modelo de regresión logística ordinal es significativa $\mathrm{p}<0,05 \mathrm{y}$ los datos se ajustan bien con una desviación de p <0,05 y la incidencia es explicada en un 55,9\% de incidencia de los factores predictores: motivación problematización o contextualización, acopio de saberes previos, propósito de la sesión, gestión y acompañamiento del aprendizaje, aplicación o transferencia de la información y evaluación en el desarrollo de las prácticas inclusivas en docentes de educación básica.

Por otro lado, se encontró que las estrategias didácticas inciden en las dimensiones del desarrollo de las prácticas inclusivas en docentes de educación básica, puesto que la razón de verosimilitud del modelo de regresión logística ordinal es significativa $\mathrm{p}<0,05 \mathrm{y}$ los datos se ajustan bien con una desviación de de $\mathrm{p}<0,05$ y se establece que los factores predictores: motivación, problematización o contextualización, recogida de saberes previos, propósito de la sesión, gestión y acompañamiento del aprendizaje, aplicación o transferencia de la información y evaluación; por lo que se establece que ante un adecuado manejo de estrategias didácticas estas inciden en cada una de las prácticas inclusivas propuestas: las estrategias de aprendizaje cooperativo, estrategias de aprendizaje dialógico, estrategias de aprendizaje servicio y comunitario, estrategias para fomentar la participación social y las estrategias integrales. 


\section{Referencias}

Álvarez, C. y González, L. (2013). Aprendizaje dialógico: una apuesta de centro educativo para la inclusión. Tabanque Revista pedagógica, 26(1), 209-224. Recuperado de https: / / dialnet. unirioja.es/servlet/articulo?codigo $=4754777$

Aubert, A., Flecha, A., García, C., Flecha, R. y Racionero, S. (2008). Aprendizaje dialógico en la Sociedad de la Información. Barcelona, España: Hipatia

Bandura, A. (1971). Social learning theory. New York: General Learning Press.

Batllé, R. (2013). El Aprendizaje. Servicio en España: el contagio de una revolución pedagógica necesaria. Madrid, España: PPC-Editorial.

Bernal, C. (2010). Metodología de la investigación. Bogotá, Colombia: Prentice Hall.

Correa, J. (2008). Prácticas de inclusión educativa y atención a la diversidad. Recuperado de https://pebaibague.weebly.com/uploads/2/3/4/3/2343628/educacion_inclusiva.pdf.

Coto, J. (2005). Guía para el Planeamiento de sesiones de enseñanza y aprendizaje. San José, Costa Rica: Instituto Nacional de Aprendizaje.

Echeita, G. (2014). Educación para la inclusión o educación sin exclusiones. Madrid, España: Narcea Ediciones.

Flores, V. J., García, I. y Romero, S. (2017). Prácticas inclusivas en la formación docente en México. Liberabit, 23(1), 39-56. Recuperado de http://dx.doi.org/https://doi. org/10.24265/liberabit.2017.v23n1.03.

García, F. y Delgado, M. (2017). Estrategias de enseñanza como respuesta a la diversidad: concepciones y prácticas del pedagogo terapéutico. Revista Nacional e Internacional de Educación Inclusiva, 10(1),103-116. Recuperado de https: / / dialnet.unirioja.es/descarga/ articulo/6049230.pdf.

García, I., Romero, S., Rubio, S., Flores, V. J., y Martínez, A. (2015). Comparación de prácticas inclusivas de docentes de servicios de educación especial y regular en México. Revista Electrónica Actualidades Investigativas en Educación, 15(3), 1-17. Recuperado de http:// www.redalyc.org/articulo.oa?id=44741347011.

García, M. y Gavira, R. (2014). Comprometiéndonos con "nuestra escuela”: un proyecto de Aprendizaje-Servicio para una formación inicial del profesorado inclusiva. Revista de Educación Inclusiva, 7(2), 69-83.

Hernández, R. y Mendoza, C. (2018). Metodología de la investigación. Las rutas cuantitativa, cualitativa y mixta. Ciudad de México: Mc Graw-Hill Education. 
Jara, O. (1989). Orientaciones teórico-prácticas para la sistematización de experiencias. Recuperado de http: / / centroderecursos.alboan.org/ebooks/0000/0788/6_JAR_ORI.pdf

León, B., Felipe, E., Iglesias, D. y Marugán, M. (2014). Determinantes en la Eficacia del Aprendizaje Cooperativo. Una experiencia en el EEES. Revista de Investigación Educativa, 32(2), 411-424. Recuperado de http://dx.doi.org/10.6018/rie.32.2.172721.

Martínez, C. (2014). Educación de calidad para todos: beneficios asociados a la atención de necesidades educativas especiales bajo el enfoque inclusivo. Santiago de Chile, Chile: Universidad de Chile.

Minedu. (2019). Los procesos pedagógicos. Recuperado de www.perueduca.pe/ documents/5080387/0/Procesos\%20pedagógicos.

Molbaek, M. (2018). Inclusive teaching strategies dimensions and agendas. International Journal of Inclusive Education, 22(1),1048-1061. Retrieved from https: / doi.org/10.1080/1360 3116.2017.1414578.

Moliner, O., Sanahuja, A. y Benet, A. (2017). Prácticas inclusivas en el aula desde la investigación acción. (tesis de maestría). Universitat Jaume, Castelló España. Recuperado de http:// dx.doi.org/10.6035/Sapientia127.

Navarro, I., González, C., López, B. y Botella, P. (2015). Aprendizaje de contenidos académicos y desarrollo de competencias profesionales mediante prácticas didácticas centradas en el trabajo cooperativo y relaciones multidisciplinares. Revista de Investigación Educativa, 33(1), 99-117. Recuperado de http://dx.doi.org/10.6018/rie.33.1.183971.

Omaña, E. y Alzolar, N. (2017). Estrategias pedagógicas para la inclusión del estudiante con discapacidad.Educ@ción en Contexto, 3(6), 83-109. Recuperado de https://dialnet. unirioja.es/descarga/articulo/6296695.pdf.

Piaget, J. (1975). Biología y conocimiento. Ciudad de México, México: Siglo XXI.

Rajadell, N. (2002). La importancia de las estrategias docentes para la resolución de conflictos en el aula. Revista Electrónica Interuniversitaria de Formación del Profesorado, 5(3), 1-7. Recuperado de http://web.archive.org/web/20041206093520/www.aufop.org/ publica $/$ reifp/articulo.asp?pid=210\&docid $=1016$.

Ramos, C. (2015). Los paradigmas de la investigación científica. Avances Psicológicos 23(1), 369-399. Recuperado de http://www.unife.edu.pe/publicaciones/revistas/ psicologia/2015_1/Carlos_Ramos.pdf.

Rodríguez, C. (2018). La diversidad funcional: estrategias didácticas en una perspectiva teórico conceptual de educación inclusiva (tesis doctoral). Universidad Católica Andrés Bello, Caracas, Venezuela.

Rogers, C. (1967). Psicoterapia y Relaciones Humanas. Madrid, España: Alfaguara. 
Rosales, A. (2004). Estrategias didácticas o de intervención docente en el área de la educación física. Revista Digital, 10(75). Recuperado de http: / /www.efdeportes.com/.

Santos, M., Lorenzo, M. y Priegue, D. (2009). Aprendizaje cooperativo: práctica pedagógica para el desarrollo escolar y cultural. MAGIS, Revista Internacional de Investigación en Educación, 2(1), 289-303. Recuperado de http: / /www.javeriana.edu.co/magis.

Santos, M. y Portaluppi, G. (2011). Educación Inclusiva. Quito, Ecuador: Ministerio de Educación.

Tichá, R. \& Abery, B. (2018). Educational practices and strategies that promote inclusion: Examples from the U.S. Social Education, 68(2), 43-62. Retrieved from doi: 10.7441/ soced.2018.06.02.03

Torrego, J. (2006). Modelo integrado de mejora de la convivencia. Estrategias de mediación y tratamiento de conflictos. Barcelona, España: Graó.

Vygotsky, L. (1979). El desarrollo de los procesos psicológicos superiores. Barcelona, España: Crítica. 